\title{
Defect Diffusion Model of InGaAs/InP Semiconductor Laser Degradation
}

\author{
Jack Jia-Sheng Huang ${ }^{1,2}$, Yu-Heng Jan ${ }^{2,1}$, Dawei Ren ${ }^{2,1}$, YiChing Hsu ${ }^{2}$, Ping Sung ${ }^{2} \&$ Emin Chou $^{2}$ \\ ${ }^{1}$ Source Photonics, 8521 Fallbrook Avenue, Suite 200, West Hills, CA 91304, USA \\ ${ }^{2}$ Source Photonics, No.46, Park Avenue $2^{\text {nd }}$ Rd., Science-Based Industrial Park, Hsinchu, Taiwan, R.O.C \\ Correspondence: Jack Jia-Sheng Huang, Source Photonics, 8521 Fallbrook Avenue, Suite 200, West Hills, CA \\ 91304, USA. Tel: 818-266-7276. E-mail: jack.huang@sourcephotonics.com
}

Received: January 6, $2016 \quad$ Accepted: January 18, $2016 \quad$ Online Published: January 29, 2016
doi:10.5539/apr.v8n1p149

\begin{abstract}
To enable high-performance fiber to the $\mathrm{x}$ (FTTx) and datacenter networks, it is important to achieve reliable and stable optical components over time. Laser diode is the essential building block of the optical components. Degradation analysis is critical for overall successful reliability design. In this paper, we study the modelling and experimental data of the InGaAs/InP laser degradation. We present a defect diffusion model that involves three propagation media ( $\mathrm{p}$-InGaAs contact, $\mathrm{p}$-InP cladding and multi-quantum wells). We propose a simple constitutive equation based on the Gauss error function to describe the defect propagation. The physical model assumes that the $\mathrm{p}$-InGaAs is the rate-limiting factor for the defect diffusion process.
\end{abstract}

Keywords: semiconductor lasers, datacenter, semiconductor technology, reliability, III-V compound semiconductor, QSFP, buried heterostructure lasers, ridge lasers

\section{Introduction}

Semiconductor devices have been widely used in commerical applications since 1980s (Grove, 1967). The technology development and growth have been driven by three main waves thus far (Oates, 2013). The first wave is by desktop PC roughly from 1980 to 2000; the second wave is by mobile phone roughly from 2000-2010; the third wave is by mobile computing since 2010. Ignited by the introduction of iPhone in 2007, the mobile computing has been showing rapid growth, driving the deployment of mega datacenters (Isaacson, 2015). Global adoption of online commerce, streaming video, social networking and cloud services has fueled the increasingly high demand of datacenters. The storage and computing requirements supported by the datacenters present new technical challenges in terms of bandwidth, transmission distance, power consumption, cost and reliability.

Due to the requirement of higher bandwidth and lower cost in the datacenters, robust design-in reliability for each component is critical for the quad small form-factor pluggable (QSFP) tranceivers (Chu, 2014). The reliability requirement is particularly stringent for the high bandwidth applications such as 40G and 100G QSFP.

For the QSFP tranceivers, distributed feedback (DFB) lasers or electro-absorption modulated lasers (EML) have been widely chosen in order to achieve low threshold current, high power and high extinction ratio (Aoki et al., 1997; Huang, 2012; Han et al., 2013). Although a number of reliability work on experimental observations was reported (Huang, 2011; Jimenez, 2003; Fukuda, 1988; Oohashi et al., 1998), there was very few defect model available in the literature (Huang, 2015).

In this paper, we study the defect diffusion model in the InGaAs/InP/InGaAsP and InGaAs/InP/InGaAlAs semiconductor lasers. We develop a physical defect diffusion model that incorparates Gauss error function as the constitutive equation. The defect diffusion processes in the propagation media of InGaAs, InP and InGaAsP/InGaAlAs quarternary quantum wells will be discussed.

\section{Experimental}

\subsection{Defect Model}

The degradation model assumed that the defect diffusion process involved three media, as shown in Fig.1. The material model for the buried heterostructure $(\mathrm{BH})$ laser consisted of the p-InGaAs as the contact layer at the top, the p-InP cladding layer in the middle and the InGaAsP MQW region at the bottom. For the ridge waveguide 
(RWG) laser, the p-InGaAs and p-InP materials were the same, and the difference was the MQW region made of InGaAlAs.

(a) BH laser

\begin{tabular}{|c|}
\hline$p-\operatorname{InGaAs}$ \\
\hline$p-\operatorname{InP}$ \\
\hline $\operatorname{InGaAsP}$ \\
\hline
\end{tabular}

(b) RWG laser

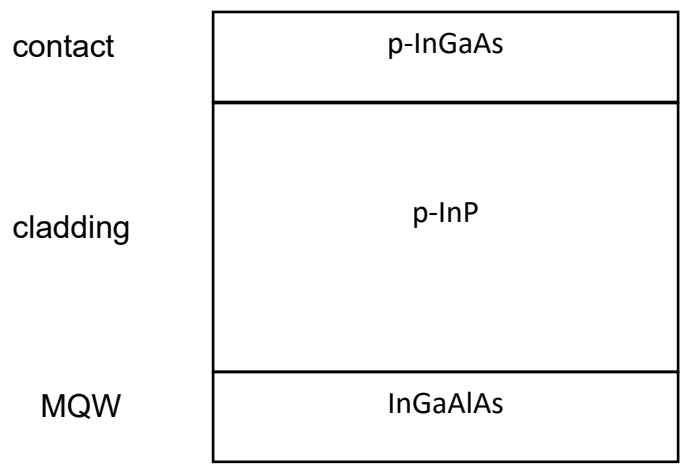

Figure 1. Materials of the defect diffusion model for (a) BH and (b) RWG laser structures. The defect propagation involves diffusion processes in the three media

(a)

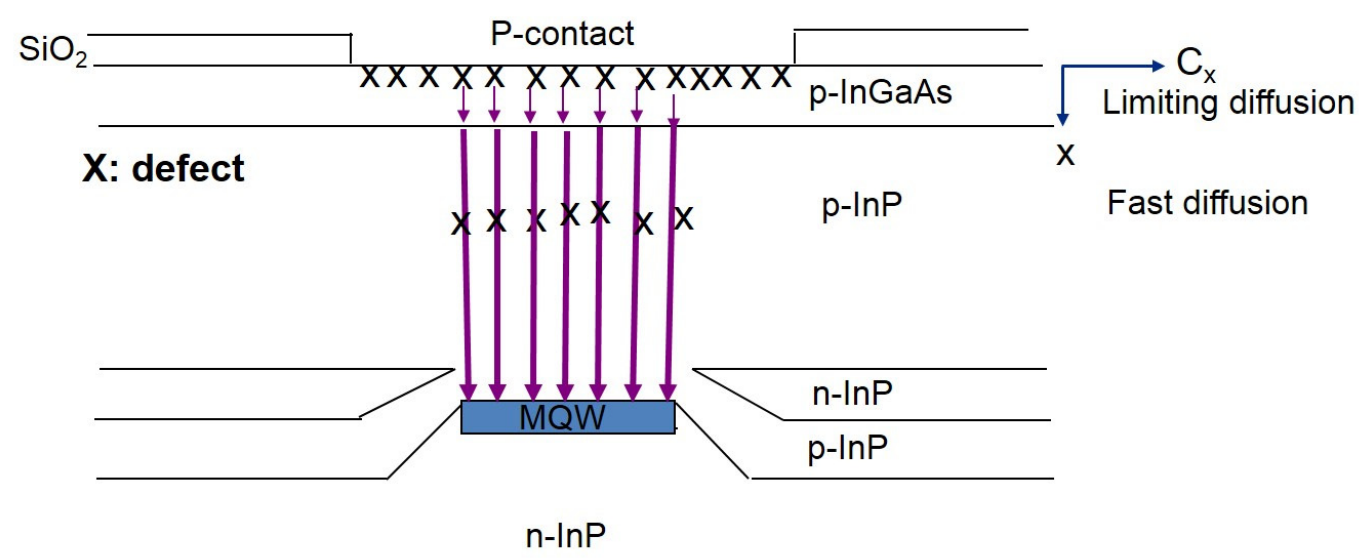

(b)

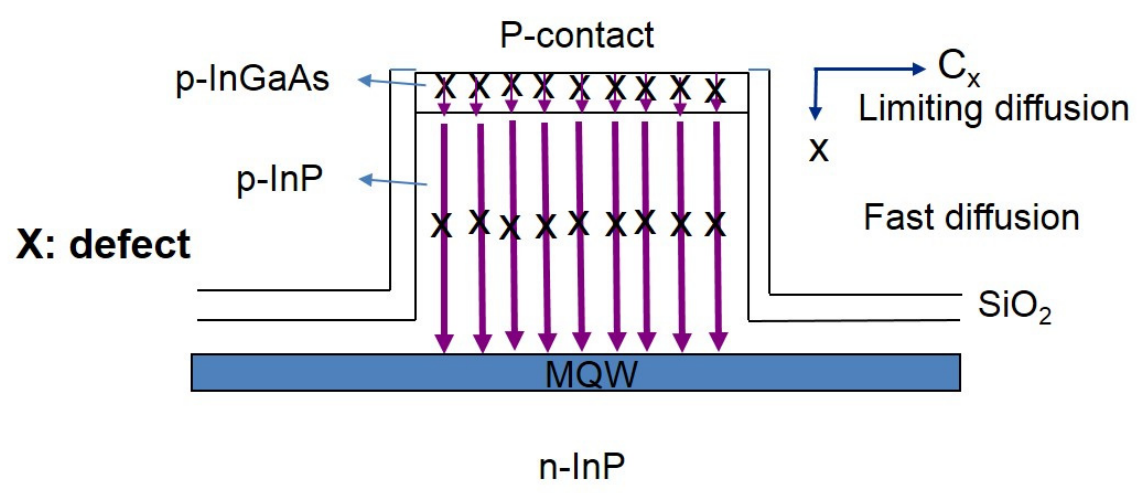

Figure 2. Schematic of defect diffusion model for (a) BH and (b) RWG lasers. The defects at the surface region are initially formed at the surface region. The defect diffusion through the $\mathrm{p}^{+}$-InGaAs layer is slow as a limiting process, as marked by the thin arrows. The defect diffusion through the underlying p-InP layer is fast, as marked by the thick arrows. Eventually, the defects enter the active region, forming DSD or DLD that are responsible for laser degradation 
Figure 2(a) and (b) show the schematics of defect diffusion model for BH and RWG lasers, respectively. The surface damaged region was initially formed at the p-doped InGaAs contact layer, marked by " $x$ ". The defect diffusion through the $\mathrm{p}^{+}$-InGaAs layer is a slow, limiting reaction, as marked by the thin arrows. The defect diffusion through the underlying p-InP layer is fast, as marked by the thick arrows. During device burn-in and aging, the defects under the driving force were propagating through the InGaAs contact, the InP and eventually into the multi-quantum well (MQW) region. The driving force included the gradient of chemical potential and the electrical current. The defect diffusion process in the InGaAs p-contact layer $\left(\mathrm{D}_{\mathrm{InGaAs}}\right)$ was slow, while the diffusion was fast in the underlying InP layer ( $\left.\mathrm{D}_{\mathrm{InP}}\right)$, shown in Equation 1 (Yu et al., 1996; Poole et al., 1995). The defects that entered the quantum well region may be in the form of point defects at the early stage. Nucleation and growth may occur over time, eventually leading to the formation of dark spot defects (DSD) and/or dark line defects (DLD).

$$
\mathrm{D}_{\mathrm{InGaAs}}<<\mathrm{D}_{\mathrm{InP}}
$$

Equation (1)

The defect diffusion model involving three media is complicated. However, we could simplify the model and focus on the analysis of the InGaAs layer due to two reasons. First, the defect diffusion in the InP is rapid and not ratelimiting. The degradation is determined by the defect propagation through the InGaAs and the defect concentration at the InGaAs/InP interface. Since the diffusivity of defects in the InGaAs was significantly lower than that in the InP (Yu et al., 1996; Poole et al., 1995), the defect diffusion in the InP would occur rapidly once the defects propagate through the InGaAs layer. Yu et al. reported fast defect diffusion in InP; the diffusion constant was in the range of $4 \times 10^{-15}$ and $1 \times 10^{-14} \mathrm{~cm}^{2} / \mathrm{sec}$ (Yu et al., 1997). Second, the defect diffusion distance is small due to the relatively thin MQW region. The degradation is typically observable when the defects reach the InP/MQW interface due to the close proximity of the $\mathrm{p} / \mathrm{n}$ junction.

In the following, we discuss the defect diffusion process in the InGaAs contact layer. We describe the diffusion by using the concentration profile based upon the Gauss error function (Smith, 1990). The concentration of the surface defect (Cs) is a function of wafer fabrication and epitaxial growth.

$$
C x=\operatorname{Cs}\left[1-\operatorname{Erf}\left(\frac{A x}{2 \sqrt{D t}}\right)\right]
$$

where $\mathrm{Cx}$ is the concentration of defect at the distance $\mathrm{x}$ from the surface, Erf is the Gauss error function, $\mathrm{A}$ is the constant, $\mathrm{D}$ is the diffusivity of defects in the InGaAs layer and $t$ is the diffusion time. The constant $\mathrm{A}$ is a function of the property of InGaAs.

The Gauss error function is the solution to Fick's second law for a non-steady-state diffusion condition.

$$
\frac{d C x}{d t}=\frac{d}{d x}\left(D \frac{d C x}{d x}\right)
$$

where the rate of compositional change with time is equal to the diffusivity times the rate of change of the concentration gradient.

For the sake of analysis, Equation 2 can be rewritten as the following:

$$
C x=C s[1-\operatorname{Erf}(K x)]
$$

where $\mathrm{K}$ is a function of constant $\mathrm{A}$, diffusivity $\mathrm{D}$ and diffusion time $\mathrm{t}$.

\subsection{Experimental Set-Up}

$$
K=\frac{A}{2 \sqrt{D t}}
$$

Equation (5)

First, the n-type indium phosphide (InP) epitaxial layer was grown on S-doped n-type InP substrate as the buffer using metal organic chemical vapor deposition (MOCVD). The active region and grating were then grown on top of the n-type buffer. For the BH, the active region was grown with the InGaAsP layers based on the sandwich structure of separate confinement heterostructure $(\mathrm{SCH}) / \mathrm{MQW} / \mathrm{SCH}$. For the RWG, the active region consisted of InGaAlAs layers based on the sandwich structure of $\mathrm{SCH} / \mathrm{MQW} / \mathrm{SCH}$. The grating layer for both $\mathrm{BH}$ and $\mathrm{RWG}$ was grown with the p-doped InGaAsP material. The DFB was defined by holographic grating, formed by lithography and wet etch. After the grating process, the grating overgrowth layer was grown. For the BH, the mesa structure was formed by wet etch and overgrown with the $\mathrm{p}$-InP and n-InP current blocking layers to minimize the leakage, followed by the p-InP cladding regrowth. For the RWG, the p-InP cladding was overgrown directly on top of the grating without mesa etch. Finally, the contact layer was formed with the heavily-doped $\mathrm{p}^{+}$-InGaAs for 
both BH and RWG. For the experimental comparison, different InGaAs layer thicknesses (0.1-0.4 micrometers) were processed.

To form the edge-emitting distributed feedback (DFB) lasers, the cleaved bars were deposited with anti-reflective (AR) and highly-reflective (HR) coatings and packaged in the traditional transistor outline cans (TO-cans) with hermetic sealed caps. The mirror coating reflectivities for the AR and HR were around $1 \%$ and $80 \%$, respectively. The TO samples were tested before and after the burn-in aging to collect the experimental degradation data.

Device characterizations such as light vs. current (LI) and optical spectrum were tested to collect the data of laser parameters. Table 1 shows the summary of the BH and RWG laser characteristics before the burn-in. For the BH, the threshold current (Ith), quantum efficiency $(\mathrm{QE})$ and output power $(\mathrm{Po})$ were superior to the RWG due to the reduction of current leakage by the $\mathrm{p}$-InP and $\mathrm{n}$-InP blocking layers.

Table 1. Summary of the BH and RWG lasers characteristics including lasing wavelength, threshold current, quantum efficiency and output power measured at $25^{\circ} \mathrm{C}$

\begin{tabular}{lllll}
\hline Laser type & Wavelength & Ith $(\mathrm{mA})$ & QE $(\mathrm{mW} / \mathrm{mA})$ & Po $(\mathrm{mW})$ at Ith+50mA \\
\hline BH & $1310 \mathrm{~nm}$ & 4.5 & 0.46 & 23.0 \\
RWG & $1270 \mathrm{~nm}$ & 9.1 & 0.41 & 20.5 \\
& $1290 \mathrm{~nm}$ & & & \\
& $1310 \mathrm{~nm}$ & & & \\
& $1330 \mathrm{~nm}$ & & & \\
\hline
\end{tabular}

\section{Results and Discussions}

Figure 3 shows the modelling results of the defect concentration as a function of the Gauss error function coefficient $(K)$. For a given surface defect concentration $\left(C_{s}\right)$, the defect concentration $\left(C_{x}\right)$ at the distance $x$ decreases with increasing coefficient where the coefficient is determined by the constant A, diffusivity $\mathrm{D}$ and diffusion time $\mathrm{t}$. In this case, we model the results with the coefficient $\mathrm{K}$ in the range of 1 to 5 . For smaller coefficient, the defect concentration becomes larger at distance $\mathrm{x}$. To interpret the physical meaning of the theoretical results shown in Figure 3, we note that the coefficient $\mathrm{K}$ based on Equation 5 is related to diffusivity (D) and diffusion time (t). For the smaller coefficient, diffusivity and/or diffusion time is larger. As a result, there is a higher density of defects that diffuse from the surface into the depth of $\mathrm{x}$. The diffusivity of defects could be affected by the quality of InGaAs epitaxial layer. Imperfections in the InGaAs such as channels could enhance the defect diffusion through the InGaAs layer.

The defect diffusion and propagation could also be enhanced by electrical stress current and non-radiative recombination. For the latter, Yu et al. (1996) showed that the generation of excess electron-hole pairs and their subsequent recombination can play an important role in the propagation of defects.

Figure 4 shows the theoretical defect concentration as a function of the surface concentration. For a given diffusivity, the defect concentration $\left(\mathrm{C}_{\mathrm{x}}\right)$ at the distance $\mathrm{x}$ increases proportionally with increasing surface defect concentration $\left(\mathrm{C}_{\mathrm{s}}\right)$. The physical meaning is that the degradation would become larger when the surface defect concentration is higher. The surface defect at the InGaAs could result from process such as reactive ion etch (RIE) during the contact opening (Huang, 2012; Morello et al., 2006) or metallic compound formation at the metal/InGaAs interface (Huang et al., 2003; Huang et al., 2005).

Based upon the aforementined modelling results of Figures 3 and 4, we can now illustrate a few case studies and correlate the modelling results with the experimental data. 


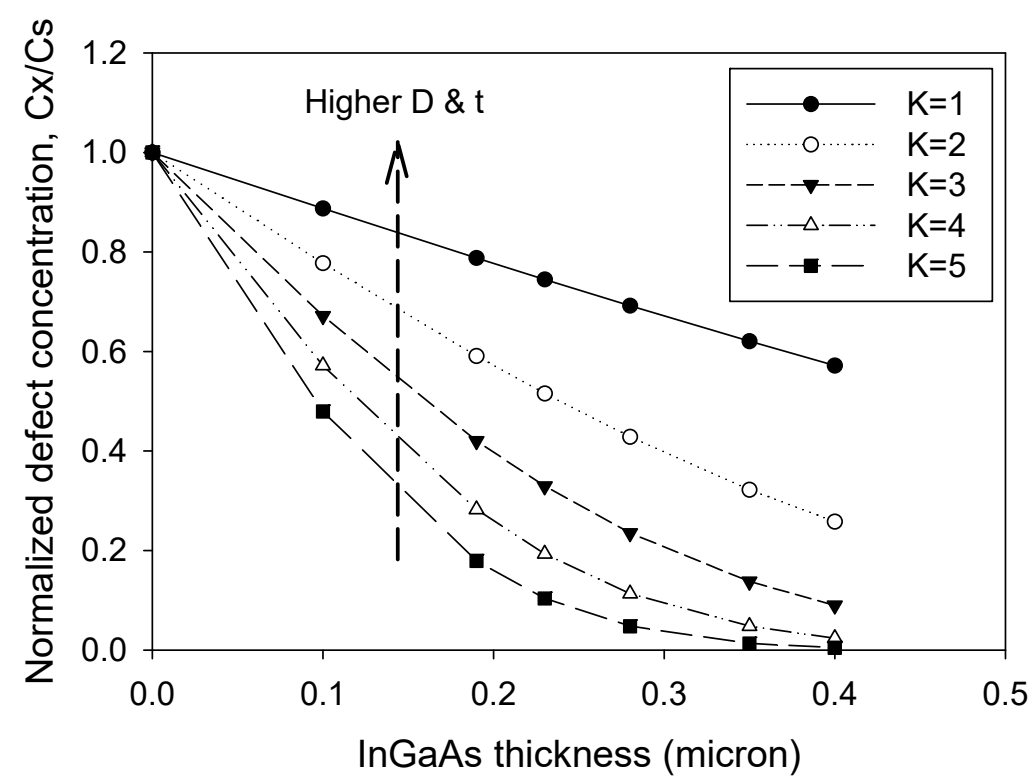

Figure 3. Theoretical defect concentration as a function of Gauss error function coefficient $(\mathrm{K})$. The smaller coefficient $\mathrm{K}$ is, the diffusivity (D) and diffusion time ( $\mathrm{t}$ ) are higher

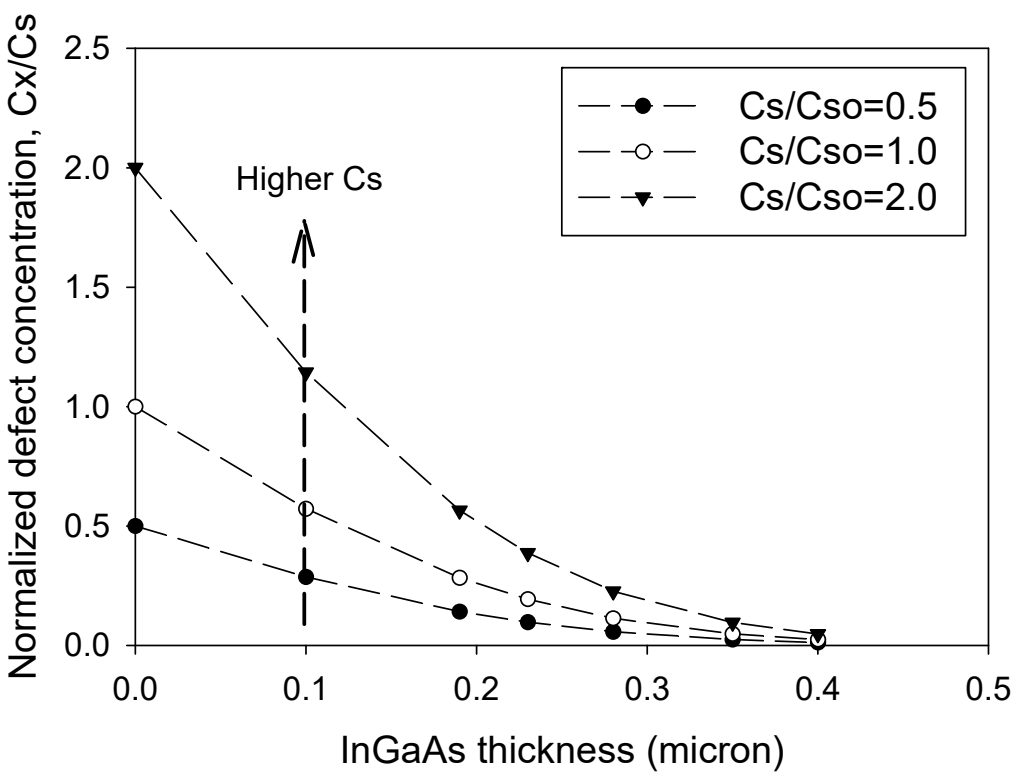

Figure 4. Theoretical defect concentration as a function of surface defect concentration $\left(\mathrm{C}_{\mathrm{s}}\right)$

\section{Case-A: InGaAs thickness dependence}

Figure 5(a) and (b) show the schematics of the defect concentraion profile as a function of the vertical axis along the InGAs, InP and MQW regions for thick InGaAs and thin InGaAs, respectively. The top surface of InGaAs corresponds to zero of the x-axis. For the thick InGaAs case in Fig.5a, the surface concentration starts as $\operatorname{Cs}(0)$ and decreases along the InGaAs layer of thickness d, following the Gauss error function. At the InGaAs/InP interface, the defect concentration is marked at Cs(d). For the thin InGaAs case in Fig.5b, the surface concentration starts as $\operatorname{Cs}(0)$ and decreases along the InGaAs layer of thickness $d / 2$, following the Gauss error function. At the InGaAs/InP interface, the defect concentration is marked at $\mathrm{Cs}(\mathrm{d} / 2)$. The defect profile shows that the defect concentration at the InGaAs/InP interface for the InGaAs thickness of $\mathrm{d} / 2$ is higher than that of the thickness $\mathrm{d}$. It 
is assumes that the defect concentration at the InGaAs/InP interface is approximately the same as that at the $\mathrm{InP} / \mathrm{MQW}$ interface due to the small concentration gradient and high diffusivity in the InP layer.

Given that the laser degradation is governed by the defect formation at the MQW region, we assume that the threshold current change $\left(\mathrm{dI}_{\mathrm{th}}\right)$ is proportional to the concentration of defects at the InP/MQW interface as follows.

(a) InGaAs thickness $=d$

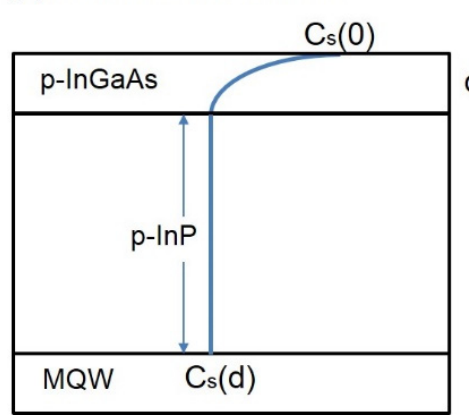

(b) InGaAs thickness $=\mathrm{d} / 2$

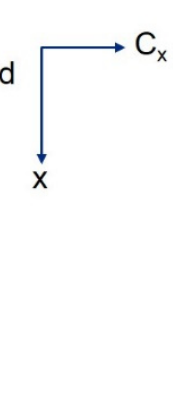

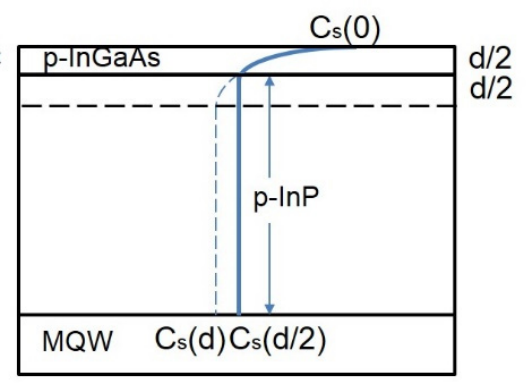

Figure 5. Schematics of the defect concentration profile as a function the vertical axis along the InGAs, InP and MQW regions for (a) thick InGaAs and (b) thin InGaAs

$$
d I t h \sim C x=C s\left[1-\operatorname{Erf}\left(\frac{A x}{2 \sqrt{D t}}\right)\right]
$$

Figure 6 shows the comparison of experimental data vs. modelling results. The theoretical curve of the concentration profile was deduced from Equation 4 to overlay with the experimental data shown by the box plot. The InGaAs thickness was measured by the scanning electron microscopy (SEM). The theoretical curve was fitted to the median of the box plot for each InGaAs thickness. The model showed the best fit when $\mathrm{K}$ was 5 . Generally, the $\mathrm{I}_{\text {th }}$ change decreased with increasing InGaAs layer thickness in the form of exponental decay. For each InGaAs thickness, the defect concentration at distance $\mathrm{x}$ could be affected by diffusivity, diffusion time and/or surface concentration. The diffusion time was the same since the burn-in time was fixed in this study. Hence, the $\mathrm{I}_{\mathrm{th}}$ change below the modelling curve implied that the diffusivity and/or surface concentration was lower and vice versa. In the following case studies, the effects of diffusivity and surface concentration will be illustrated and discussed.

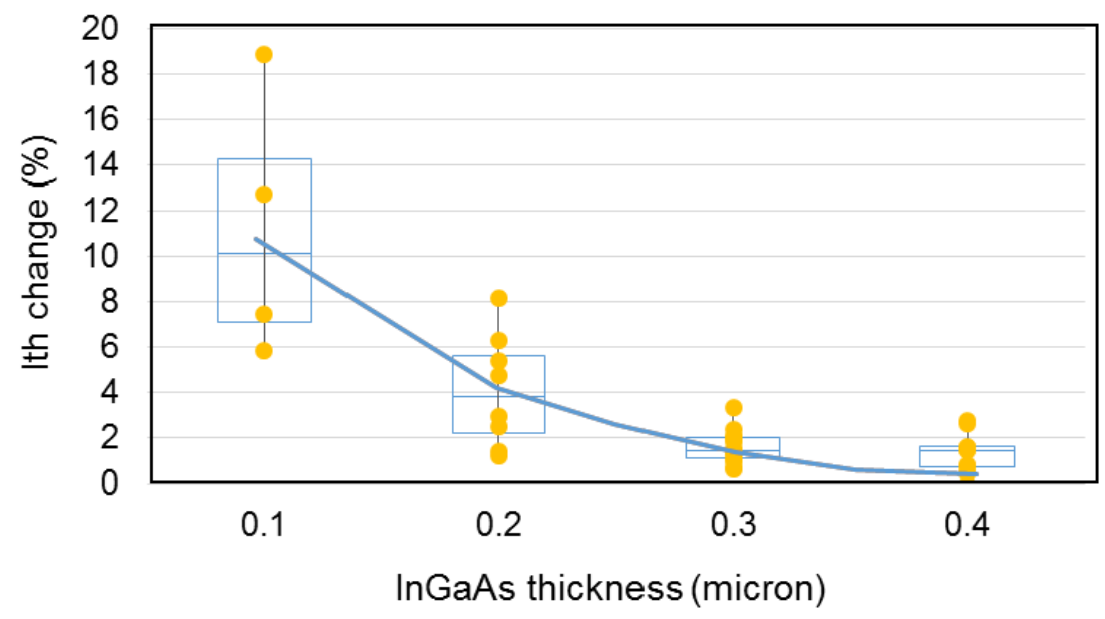

Figure 6. Modelling vs. experimental degradation of InGaAs/InP lasers. The plot shows the experimental data overlaid with the modelling results 


\section{Case-B: Diffusivity Dependence}

Figure 7 shows the schematic of the defect concentraion profile as a function of diffusivity. For a given surface defect concentration, the defect concentration at the InGaAs/InP increases with increasing diffusivity. Again, the defect concentration at the InGaAs/InP interface is approximately the same as that at the InP/MQW interface due to the small concentration gradient. Hence, the defect profile associated with higher diffusivity shows higher defect concentration at the InGaAs/MQW interface, resulting in faster degradation and larger Ith change.

One example for the high diffusivity is the RWG structure with RIE contact etch. The defect diffusion rate in the InGaAs could be enhanced by the RIE-induced plasma damage (Huang, 2015; Morello et al., 2006).The RIE damage may degrade the property of regrowth and enhance the diffusion of the surface defects. The other example for the high diffusivity is the BH structure with a thin buffer between the epitaxial quantum well region and the substrate. When the buffer layer underneath the active region is thin enough to allow the interaction between the mesa etch and the substrate, more defects are likely to be formed during the mesa etch process (Huang, 2012).We postulate that the defects originating from the etched mesa might be building up during the progression of blocking and final epitaxial regrowth. The more deficient InP regrowth may in turn lead to more deficient InGaAs layer that could enhance the diffusion during aging. This process is called "bottom up" defect propagation mechanism.

There are several remedies to minimize the defect diffusivity and laser degradation. One example of the low diffusivity is the RWG incorporated with wet etch in the contact opening. The wet etch process could eliminate the plasma damage during the RIE etch. The other instance is the BH with a thick buffer layer in order to prevent the punchthrough from the mesa etch into the substrate. The thick buffer layer could help improve the quality of the regrowth and suppress the diffusion of the defects.

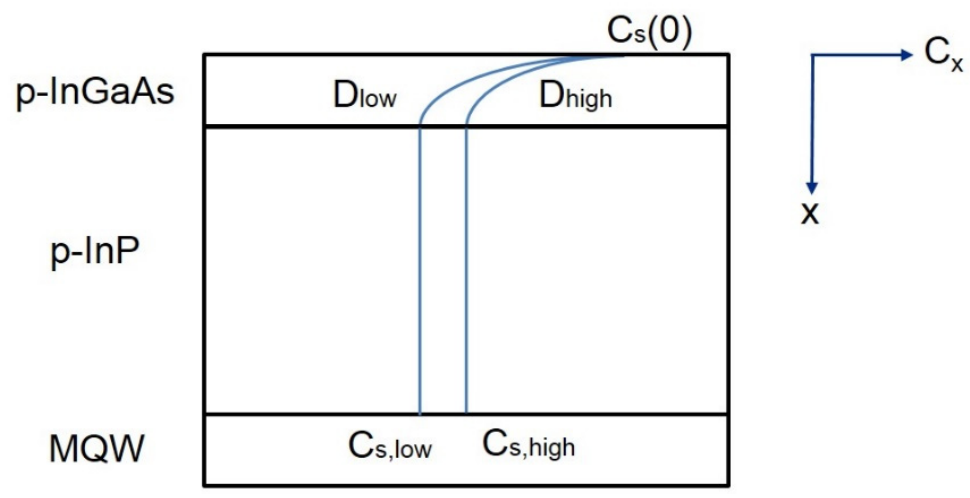

Figure 7. Schematic of the defect concentration profile as a function of diffusivity along the vertical axis. The high diffusivity in the InGaAs layer would lead to higher defect concentration at the InGaAs/InP interface and at the InP/MQW interface

\section{Case-C: Surface Concentration Dependence}

Figure 8 shows the schematic of the defect concentraion profile as a function of surface defect. Given the same diffusivity, the higher surface defect concentration would lead to higher defect density at the InGaAs/InP and InP/MQW interfaces. Hence, the defect profile associated with higher surface defect density shows higher defect concentration at the InGaAs/MQW interface, resulting in greater degradation and larger Ith change.

One example of the high surface defect concentration is the RWG structure with RIE contact etch where the surface defect could be accumulated at the InGaAs layer as a result of plasma damage from RIE. The accumulation and buildup of the surface defects are likely confined in the InGaAs layer before the laser devices are subjected to the current stress due to the lower defect diffusivity of the InGaAs layer.

The other example of high surface defect density is the BH structure with a thin buffer layer between the active region and the substrate. The surface defect is higher resulting from the "bottom up" defect mechanism. With the origin of mesa etch defects, more defects are accumulating during the regrowth from the bottom. The surface defect density is thereby expected to be higher for such BH mesa structure. 


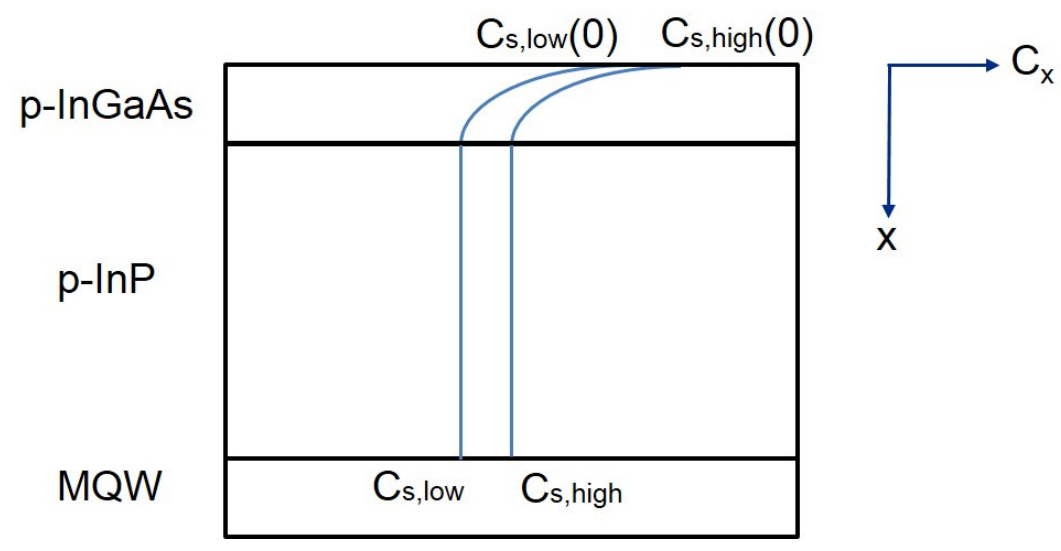

Figure 8. Schematic of the defect concentration profile as a function of surface defect density along the vertical axis. The high surface concentration would lead to higher defect concentration at the InGaAs/InP interface and at the InP/MQW interface

Besides the aforementioned Gauss error function, the other alternative model that could describe the defect propagation in the InGaAs layer is the Wentzel-Kramers-Brillouin (WKB) method (Landau et al., 1958; Shankar, 1994). In quantum physics, the WKB method was widely used to explain the transmission of electron tunneling through the energy barrier. In the defect diffusion process through the InGaAs and InP layers, the defect and InGaAs could be viewed as the electron and barrier, respectively. The "tunneling" probability of a defect through the InGaAs barrier can be given by

$$
T_{t}=\exp \left[-2 \int_{0}^{d}|k(x)| d x\right.
$$

where $T_{t}$ is the tunneling probability, $x$ is the diffusion distance and $d$ is the thickness of the InGaAs. The details of the WKB modeling would be an interesting subject for future study.

\section{Conclusion}

We presented a defect diffusion model to account for the degradation of InGaAs/InP lasers. The model assumed the InGaAs layer being the rate-limiting factor due to its low diffusivity. We proposed a simple constitutive equation based upon Gauss error function to describe the defect diffusion process. The model showed that the defect concentration at the InGaAs/InP interface increased with decreasing InGaAs thickness. It was consistent with the experimental observation where the $\mathrm{I}_{\mathrm{th}}$ change was found to increase generally with decreasing InGaAs thickness.

The modelling also showed that the variation in the $\mathrm{I}_{\text {th }}$ change for each InGaAs thickness was related to the surface defect concentration $\left(\mathrm{C}_{\mathrm{s}}\right)$ and diffusivity (D). For the latter, the diffusivity of the InGaAs and InP may be affected by the underlying MQW materials processing. For the BH laser with the InGaAsP MQW, the defects formed during the mesa etch were likely to build from bottom up during the subsequent regrowth of InP and InGaAs layers. The more defective overgrown layers would enhance the diffusivity of the defects. For the RWG laser with the InGaAlAs MQW, the pre-existing defect density was lower because there was no mesa etch prior to the overgrowth. For the former, the surface defect concentration may be influenced by the process steps such as contact cleaning and RIE etch. The reliability sensitivity to the InGaAs thickness was higher in the BH laser likely due to the higher pre-existing defect density.

\section{References}

Aoki, M., Komori, M., Tsuchiya, T., Sato, H., Nakahara, K., \& Uomi, K. (1997). InP- based reversed-mesa ridgewaveguide structure for high-performance long-wavelength laser diodes, IEEE J. Selected Topics Quantum Electron. Vol. 3, no. 2, p.672-683. http://dx.doi.org/10.1109/2944.605720

Chu, S. (2014). Source Photonics introduces industry-first 100G LR4 transceiver in QSFP28 form factor for datacenter application. San Francisco, CA: Optical Fiber Communications Conference.

Grove, A. S. (1967). Physics and Technology of Semiconductor Devices. New York, NY: John Wiley \& Sons Inc. 
Han, Y. T., Kwon, O. K., Lee, D. H., Lee, C. W., Lee, Y. A., Shin, J. U., ... Baek, Y. (2013). A cost-effective 25$\mathrm{Gb} / \mathrm{s}$ EML TOSA using all-in-one FPCB wiring and metal optical bench (pp. 26962-26971). Optics Express. http://dx.doi.org/10.1364/OE.21.026962

Huang, J. S. (2011). Design-in reliability of modern distributed feedback (DFB) InP lasers: Can we meet up the stringent wavelength-division multiplex (WDM) requirement? Montreal, Quebec, Canada: IEEE Photonic Society Summer Topical (pp. 89-90). http://dx.doi.org/10.1109/phosst.2011.6000059

Huang, J. S. (2012). Design-in reliability for modern wavelength-division-multiplex (WDM) distributed feedback (DFB) InP lasers. Appl. Physics. Research, 4(2), 15-28. http://dx.doi.org/10.5539/apr.v4n2p15

Huang, J. S. (2015). Reliability of optoelectronics. In J. Swingler (Ed.), Reliability characterisation of electrical and electronic systems (Chapter 6). Cambridge, United Kingdom: Woodhead Publishing. http://dx.doi.org/10.1016/b978-1-78242-221-1.00006-x

Huang, J. S., \& Vartuli, C. B. (2003). Scanning transmission electron microscopy study of $\mathrm{Au} / \mathrm{Zn} / \mathrm{Au} / \mathrm{Cr} / \mathrm{Au}$ and $\mathrm{Au} / \mathrm{Ti} / \mathrm{Pt} / \mathrm{Au} / \mathrm{Cr} / \mathrm{Au}$ contacts to p-type InGaAs/InP. J. Appl. Phys., 93(9), 5196-5200. $\mathrm{http}: / / \mathrm{dx}$. doi.org/10.1063/1.1565187

Huang, J. S., Nguyen, T., Hsin, W., Aeby, I., Ceballo, R., \& Krogen, J. (2005). Reliability of etched-mesa buried heterostructure semiconductor lasers. IEEE Trans. Device Mater. Reliab., 5(4), 665-674. http://dx.doi.org/10.1109/TDMR.2005.860562

Isaacson, W. (2015). Steve Jobs. New York, NY: Simon \& Schuster.

Jimenez, J. (2003). Laser diode reliabillity: crystal defects and degradation modes. C.R. Physique, 4, 663-673. http://dx.doi.org/10.1016/S1631-0705(03)00097-5

Landau, L. D., \& Lifshitz, E. M. (1958). Quantum Mechanics. Reading, MA: Addison- Wesley.

Fukuda, M. (1988). Lasers and LED reliability update. J. Lightwave Tech., 6(10), 1488-1495.

Morello, G., Quaglic, M., Meneghini, G., Papuzza, C., \& Kompocholis, C. (2006). Reactive ion etching induced damage evaluation for optoelectronic device fabrication. J. Vac. Sci. Tech. B, 24(2), 756-761. http://dx.doi.org/10.1116/1.2181576

Oates, A. S. (2013). Will Reliability limit Moore's law? Lake Tahoe, NV: Keynote, International Integrated Reliability Workshop.

Oohashi, H., Fukuda, M., Kondo, Y., Wada, M., Tohmori, Y., Sakai, Y., ... Itaya, Y. (1998). Reliability of 1300$\mathrm{nm}$ spot-size converter integrated laser diodes for low-cost optical modules in access networks. J. Lightwave Tech., 16(7), 1302-1307. http://dx.doi.org/10.1109/50.701409

Poole, P. J., Charbonneau, S., Aers, G. C., Jackman, T. E., Buchanan, M., Dion, M., ... Mitchell, I. V. (1995). Defect diffusion in ion implanted AlGaAs and InP: Consequences for quantum well intermixing. J. Appl. Phys., 78(4), 2367-2371. http://dx.doi.org/10.1063/1.360157

Shankar, R. (1994). Principles of Quantum Mechanics (Chap. 16.). New Haven, CT: Springer. http://dx.doi.org/10.1007/978-1-4757-0576-8

Smith, W. F. (1990). Principles of Materials Science and Engineering (Chap. 4). New, York: McGraw-Hill.

Yu, D. G., Chen, C. H., Holmes, A. L., \& DenBaars, S. P. (1996). Photoluminescence studies n radiation enhanced diffusion of dry-etched damage in GaAs and InP materials. J. Vac. Sci. Technol. B, 14(6), 3684-3687. http://dx.doi.org/10.1116/1.588750

Yu, D. G., Chen, C. H., Holmes, A. L., DenBaars, S. P., \& Hu, E. L. (1997). Role of defect diffusion in the InP damage profile. J. Vac. Sci. Technol. B, 15, 2672. http://dx.doi.org/10.1116/1.589706

\section{Copyrights}

Copyright for this article is retained by the author(s), with first publication rights granted to the journal.

This is an open-access article distributed under the terms and conditions of the Creative Commons Attribution license (http://creativecommons.org/licenses/by/3.0/). 